\title{
Psilocybin therapy increases cognitive and neural flexibility in patients with major depressive disorder
}

\author{
Manoj K. Doss (iD ${ }^{1,2}$, Michal Považan ${ }^{3}$, Monica D. Rosenberg (D) $^{4}$, Nathan D. Sepeda ${ }^{1,2}$, Alan K. Davis ${ }^{2,5}$, Patrick H. Finan ${ }^{1}$, \\ Gwenn S. Smith (iD ${ }^{1,3}$, James J. Pekar ${ }^{3,6}$, Peter B. Barker $\mathbb{D}^{3}$, Roland R. Griffiths $\mathbb{D}^{1,2,7}$ and Frederick S. Barrett ${ }^{1,2}$
}

(c) The Author(s) 2021

Psilocybin has shown promise for the treatment of mood disorders, which are often accompanied by cognitive dysfunction including cognitive rigidity. Recent studies have proposed neuropsychoplastogenic effects as mechanisms underlying the enduring therapeutic effects of psilocybin. In an open-label study of 24 patients with major depressive disorder, we tested the enduring effects of psilocybin therapy on cognitive flexibility (perseverative errors on a set-shifting task), neural flexibility (dynamics of functional connectivity or dFC via functional magnetic resonance imaging), and neurometabolite concentrations (via magnetic resonance spectroscopy) in brain regions supporting cognitive flexibility and implicated in acute psilocybin effects (e.g., the anterior cingulate cortex, or ACC). Psilocybin therapy increased cognitive flexibility for at least 4 weeks post-treatment, though these improvements were not correlated with the previously reported antidepressant effects. One week after psilocybin therapy, glutamate and $\mathrm{N}$-acetylaspartate concentrations were decreased in the ACC, and AFC was increased between the ACC and the posterior cingulate cortex (PCC). Surprisingly, greater increases in dFC between the ACC and PCC were associated with less improvement in cognitive flexibility after psilocybin therapy. Connectome-based predictive modeling demonstrated that baseline dFC emanating from the ACC predicted improvements in cognitive flexibility. In these models, greater baseline dFC was associated with better baseline cognitive flexibility but less improvement in cognitive flexibility. These findings suggest a nuanced relationship between cognitive and neural flexibility. Whereas some enduring increases in neural dynamics may allow for shifting out of a maladaptively rigid state, larger persisting increases in neural dynamics may be of less benefit to psilocybin therapy.

Translational Psychiatry (2021)11:574; https://doi.org/10.1038/s41398-021-01706-y

\section{INTRODUCTION}

Recently, classic psychedelics (serotonin $2 \mathrm{~A}$ or $5-\mathrm{HT}_{2 \mathrm{~A}}$ agonists such as psilocybin, lysergic acid diethylamide or LSD, and $\mathrm{N}, \mathrm{N}$-dimethyltryptamine or DMT) have shown potential efficacy for treating a variety of psychiatric disorders [1-6], including major depressive disorder (MDD). Whereas typical antidepressant treatments such as selective serotonin reuptake inhibitors (SSRIs) can take weeks to months to take effect, two administrations of psilocybin coupled with extensive psychotherapy appear to have rapid and enduring antidepressant effects (e.g., lasting weeks to months after the acute effects $[2,3]$ and at least as effective as a SSRI [7]). Despite these striking effects of psychedelic therapy, the cognitive and neural mechanisms underlying their enduring efficacy are poorly understood.

A potential transdiagnostic neuropsychological mechanism that may be targeted by psychedelic therapy is cognitive flexibility. Cognitive flexibility is broadly defined as the ability to adaptively switch between different cognitive operations in response to changing environmental demands, and it is typically measured as perseveration on prior rules in set shifting or rule switching tasks (for review, see [8]). Impairments in cognitive flexibility have been found in depression [9, 10], as well as obsessive-compulsive disorder [11] and substance use disorders [12], which are disorders that may also be amenable to psychedelic therapy $[1,5,6]$. Cognitive flexibility deficits may precede the onset of other symptoms in depression [13], have been identified across the lifespan in patients with depression [14, 15], and may represent an endophenotype for depression [16]. Although cognitive flexibility may not always be directly related to global measures of symptomology for a given disorder [17], the capacity to readily shift between different mental states could especially be useful in the context of psychotherapy [18]. Furthermore, the related concept of psychological flexibility has been found to mediate the relationship between acute psychedelic effects and improvements in depression and anxiety [19]. Antidepressant treatments including ketamine [20], SSRIs [21-23], duloxetine (a serotonin and norepinephrine reuptake inhibitor) [17], and repetitive transcranial magnetic stimulation [22] have been found to improve cognitive flexibility, perhaps by driving plasticity of the hippocampus [24, 25], a structure implicated in depression [26] and flexible behavior [27]. Nevertheless, many patients remain treatment-refractory for cognitive deficits [28, 29], necessitating research on novel treatments.

\footnotetext{
${ }^{1}$ Department of Psychiatry and Behavioral Sciences, Johns Hopkins University School of Medicine, Baltimore, USA. ${ }^{2}$ Center for Psychedelic \& Consciousness Research, Johns Hopkins University School of Medicine, Baltimore, USA. ${ }^{3}$ Department of Radiology and Radiological Science, Johns Hopkins University School of Medicine, Baltimore, USA. ${ }^{4}$ Department of Psychology, University of Chicago, Chicago, USA. ${ }^{5}$ College of Social Work, The Ohio State University, Columbus, USA. ${ }^{6}$ F.M. Kirby Research Center for Functional

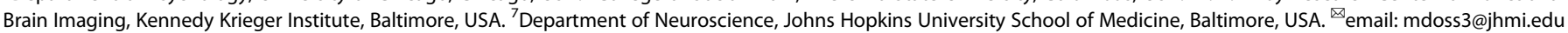


Although it is unclear what the enduring effects of psychedelic therapy are on cognitive flexibility in humans, the acute effects of $5-\mathrm{HT}_{2 \mathrm{~A}}$ receptor modulation on cognitive flexibility are mixed. Blockade of $5-\mathrm{HT}_{2 \mathrm{~A}}$ receptors has been found to both impair $[30,31]$ and enhance cognitive flexibility in animal models [32-34]. In contrast, activation of $5-\mathrm{HT}_{2 \mathrm{~A}}$ receptors with psychedelic drugs has been found to acutely impair cognitive flexibility in humans [35] and impair or have no impact on cognitive flexibility in animals [36, 37]. Moreover, psychedelics also activate the $5-\mathrm{HT}_{2 \mathrm{C}}$ receptor, and the relationship between modulation of this receptor and cognitive flexibility is complex. In animal models, $5-\mathrm{HT}_{2 \mathrm{C}}$ blockade alone has been found to enhance $[30,38]$ or have no impact cognitive flexibility [34]. However, one study found that $5-\mathrm{HT}_{2} \mathrm{C}$ blockade during co-administration of a psychedelic impaired cognitive flexibility [36] when the administration of the psychedelic alone had no impact, suggesting that coactivation of the $5-\mathrm{HT}_{2} \mathrm{C}$ receptor may be protective against impairments in cognitive flexibility from $5-\mathrm{HT}_{2 \mathrm{~A}}$ activation. Together, these findings suggest that acute activation or blockade of receptors targeted by psychedelics can bidirectionally modulate cognitive flexibility, though it remains unclear whether any of these effects extend into the days following psychedelic administration.

Activity of the anterior cingulate cortex (ACC) and its interactions with other regions $[39,40]$ are known to support cognitive flexibility, and single units of the ACC are involved in search for new rules to promote adaptive behavior [41]. Several pieces of evidence suggest that psychedelics modulate the activity of the ACC. In humans, psychedelics have been found to acutely increase ACC activity [42-44] and task-free static functional connectivity (sFC; the temporal coupling between the activity of two regions) between the default mode and salience networks [45-47], the ACC being a major hub of the latter. Increased sFC between the default mode and salience networks [48] and between the ACC and posterior cingulate cortex (PCC; [49]) has also been observed 1 day after DMT administration (in the orally active form, ayahuasca). Like the ACC, activity and SFC of the PCC are consistently altered during acute effects of classic psychedelics [46, 50-52], and concentrations of glutamate and $\mathrm{N}$-acetylaspartate (NAA) in the PCC were reduced 1 day post-DMT administration [49]. Finally, SFC between the PCC and the subgenual cingulate, a region ventral to the $A C C$, was increased 1 day after psilocybin therapy in patients with MDD [53]. The subgenual cingulate is a major target in the treatment of depression [54], and reduced SFC between the subgenual cingulate and ACC has been observed in MDD and is associated with emotional rigidity [55].

Recently, several studies have suggested that psychedelics induce neural plasticity [56-60] (for reviews, see [61, 62]). With markers of neural plasticity difficult to assess in humans, a potential proxy for ongoing shifts in synaptic weighting within and across brain regions may come from measures of neural flexibility in functional magnetic resonance imaging (fMRI) data (i.e., signal variability over time). Different measures of neural flexibility, especially those involving the salience network [63], have been associated with cognitive flexibility $[8,64]$, and neural flexibility of the ACC, as well as the PCC, has been found to be decreased in MDD $[65,66]$. Moreover, psychedelics and other hallucinogens have been found to acutely increase various measures of variance and entropy in brain activity, especially in regions of the salience network including the ACC ([67-71]; though decreases have also been reported [46, 71, 72]). Although the durability of these effects is unclear in patients with MDD, one small study of 12 healthy adults did not find enduring modulation of neural flexibility or ACC function 1 week and 4 weeks after a single dose of psilocybin [73].

Here, we utilized both hypothesis-driven and data-driven approaches of multi-modal brain imaging measurements to examine the enduring effects of psilocybin therapy on cognitive and neural flexibility in patients with MDD. Specifically, we examined the relationship between changes in depression, cognitive flexibility, and both sFC and the dynamics of functional connectivity ( $\mathrm{dFC}$; variance in the timeseries of moment-to-moment sFC as measured with fMRI) following psilocybin therapy. In addition, we collected magnetic resonance spectroscopy (MRS) data to characterize psilocybin-induced changes in neurometabolite concentrations in the ACC and hippocampus, regions involved in cognitive flexibility $[24,25,27,39-41]$ with abnormal glutamate and NAA concentrations in depression that are responsive to antidepressant treatments [74-76]. As the MRI was performed at $7 \mathrm{~T}$, there was dropout in the fMRI signal in some parts of the brain including the hippocampus. Thus, we narrowed our focus of sFC and $\mathrm{dFC}$ to interactions involving the ACC and PCC.

\section{METHODS \\ Participants}

Detailed participant information was previously published [3]. Twenty-four participants (eight males) aged 24-59 years $(M=39.83, S D=12.23$ ) with MDD ( $\geq 17$ on the GRID-Hamilton Depression Rating Scale or GRID-HAMD) completed neuroimaging and/or cognitive tasks (see specific measures for missing data) in an open-label clinical trial (ClinicalTrials.gov Identifier: NCT03181529). This sample size was chosen based on previous work that found a large effect size on the GRID-HAMD [4] (i.e., the primary outcome of this clinical trial). Participants were recruited through advertisements and word-of-mouth referrals. Screening included internet surveys, phone interviews, and in-person medical and psychiatric assessments. Exclusion criteria included current antidepressant medication, substantial lifetime use (>10 total) or recent use (past 6 months) of ketamine or classic psychedelics, a current significant medical condition, personal or family history (first or second degree) of psychotic or bipolar disorders, moderate or severe alcohol or other drug use disorder (including nicotine) in the past year, standard fMRI contraindications (e.g., left-handed, incompatible medical devices), and for women, being pregnant or nursing. This study was approved by the Johns Hopkins Medicine Institutional Review Board, and all participants provided informed consent.

\section{Psilocybin therapy}

Detailed information regarding the full treatment procedure can be found elsewhere [3]. Thirteen and 11 participants were randomly assigned (via urn randomization) to an immediate treatment and delayed treatment group, respectively. After screening, participants in the immediate group completed baseline clinical, cognitive, and neuroimaging measurements followed by $\sim 8 \mathrm{~h}$ of preparatory therapy sessions conducted over 2 weeks to build rapport with research personnel. Following these preparatory sessions, participants attended two psilocybin sessions separated by $\sim 1.6$ weeks. A moderately high dose $(20 \mathrm{mg} / 70 \mathrm{~kg})$ and a high $(30 \mathrm{mg} /$ $70 \mathrm{~kg}$ ) dose of psilocybin in opaque gelatin capsules were orally consumed at the first and second sessions, respectively. During these sessions, participants lay on a couch in a comfortable, dimly lit room while wearing eyeshades and listening to music (mostly Western art music) through headphones. Throughout these sessions, research personnel remained in the room at all times to respond to participants' needs, though participants were encouraged to remain in a supine position and direct their attention inward. After each psilocybin session, participants attended follow-up meetings to discuss their experiences.

In order to account for non-treatment-related changes in depression (e.g., interacting with research personnel) and performance on cognitive tasks (i.e., practice effects), the 11 participants in the delayed group completed clinical and cognitive (but not neuroimaging) measurements after screening (pre-delay assessments) and were then monitored weekly through in-person visits and telephone calls during an 8-week delay. At the end of this delay period, participants completed a second set of clinical and cognitive measurements and their first neuroimaging measurements (baseline assessments) followed by the same preparatory, psilocybin, and follow-up sessions administered to the immediate treatment group.

\section{Clinical and cognitive measures}

Depression was assessed with the 17-item GRID version of the HAMD [77] administered by blinded clinician raters via telephone at screening, 1 week post-treatment, and 4 weeks post-treatment. In the delayed control group, the GRID-HAMD was also assessed after 5 and 8 weeks into the delay period. 

Depression

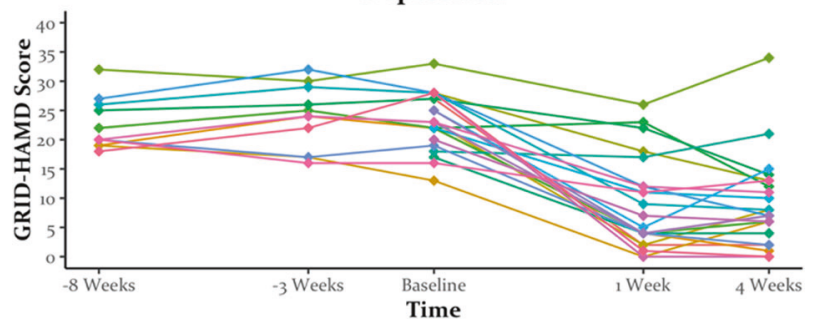

b

Effects of Psilocybin Therapy on

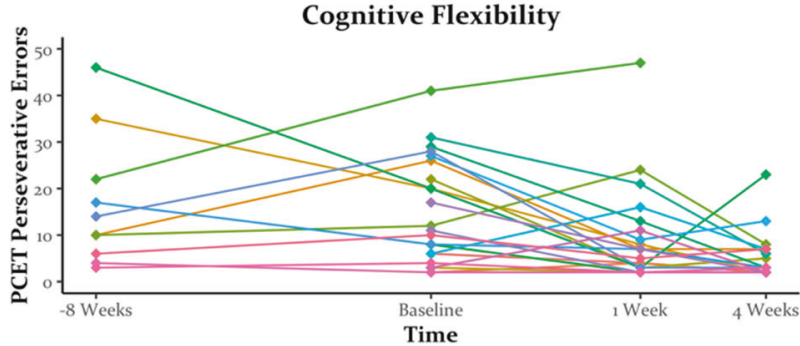

Fig. 1 The effects of psilocybin therapy on depression and cognitive flexibility. Depression symptomology (a) as measured by the GRID-Hamilton Depression Rating Scale (GRID-HAMD; also reported in [3]) and cognitive flexibility (b) as measured by perseverative errors on the Penn Conditional Exclusion Test (PCET) were improved from pre- to post-psilocybin therapy. These changes were not found between repeated tests pre-psilocybin therapy in the delayed group (i.e., between -8 weeks and Baseline time points). Each line color represents a unique participant, and the mapping of individual colors to unique participants remains consistent across all figures in which individual participant data are plotted.

Notable effects of psilocybin therapy were limited to the Penn Conditional Exclusion Test (PCET; [78]), and descriptions and analyses of the other two tasks can be found in the Supplementary Information (Sl; Table S1). The PCET is a set-shifting measure of cognitive flexibility modeled after the Wisconsin Card Sorting Task [79], but the PCET has multiple validated alternate test forms with different stimuli to support repeated measurements. Participants completed a different test form at every visit, and the order of these test forms was counterbalanced across participants. On each trial of the PCET, four figures were simultaneously presented, and participants were required to select the figure that was different from the other three based on one of three criteria (Version 1: line thickness, shape, size; Version 2: location of shape within stimulus box, color, type of shape within stimulus box; Version 3: angle of lines within stimulus box, dashed versus solid lines within stimulus box, and presence or absence of a border on stimulus box; Version 4: sharp or rounded edges, angle of stimulus box, filled or empty stimulus box). Once a figure was selected, participants were presented with immediate feedback ("correct" or "incorrect"). After 10 consecutive correct trials or after 48 total trials, the criterion for a correct response changed until another 10 consecutive correct trials or 48 total trials was reached after which the criterion was changed one last time (followed by a final round of 10 consecutive correct or 48 total trials). Perseverative errors were defined as the number of instances in which three incorrect responses are made based on a previous rule, and they are thought to reflect less cognitive flexibility (or cognitive rigidity). See SI for descriptions and data of other responses. One participant (female, delayed group) refused to complete cognitive tasks, and another participant (female, immediate group) was excluded for looking up the rules to the PCET after the first test (resulting in $N=22$ ). Due to a technical error, data from 4 weeks post-treatment was missing from an additional participant (female, delayed group).

\section{Imaging parameters and preprocessing}

Approximately 4 weeks before the first psilocybin session and 1 week after the second psilocybin session (on the same days as cognitive testing) participants were scanned at $7 \mathrm{~T}$ (Philips Achieva, Best, The Netherlands) using a 32-channel head coil (Nova Medical, Wilmington, MA). Measurements at each scanning session included a T1-weighted structural MPRAGE $\left(T R / T E=4100 / 1.86 \mathrm{~ms}\right.$, flip angle $=7^{\circ}$, acquisition matrix $=220 \times 220 \mathrm{~mm}$, voxel size $=1 \mathrm{~mm}^{3}$ ), an echo-planar scan with an emotional processing task (to be reported elsewhere), an echo-planar scan to measure task-free (eyes open) blood-oxygenation level-dependent (BOLD) fMRI (355 TRs, TR/ $\mathrm{TE}=2000 / 22 \mathrm{~ms}$, flip angle $=60^{\circ}$, acquisition matrix $=192 \times 192 \mathrm{~mm}$, inplane resolution $=2.5 \times 2.5 \mathrm{~mm}$, slice thickness $=3 \mathrm{~mm}$, SENSE acceleration factor $=3,54$ axial-oblique slices parallel to the anterior-posterior commissure line), and stimulated echo acquisition mode (STEAM) short-TE ${ }^{1} \mathrm{H}$-MRS scans (TR/TE $/ \mathrm{TM}=3000 / 33 / 14$, acquisition time $=6 \mathrm{~min} 30 \mathrm{~s}$, NT $=$ 128 , bandwidth $=5000 \mathrm{~Hz}$, VAPOR water suppression; water-unsuppressed spectra were acquired with similar parameters and NT $=2$ ) of the ACC (voxel size $=30 \times 20 \times 20 \mathrm{~mm}^{3}$ ), left hippocampus (voxel size $=35 \times 15 \times$ $15 \mathrm{~mm}^{3}$ ), and right hippocampus (voxel size $=35 \times 15 \times 15 \mathrm{~mm}^{3}$; see Fig. $\mathrm{S} 1$ for example of spectroscopic voxel placement). Three participants (one male, all immediate group) did not complete at least one imaging session, and one participant (male, delayed group) did not complete a resting state scan. In addition, one participant (male, delayed group), three participants (one male, delayed group and two females, immediate group), and two participants (both females, one immediate group) had poor signal (spectral abnormalities, signal-to-noise $<5$, or a Cramer-Rao Lower Bound standard deviation $>20$ for glutamate or NAA) in at least one of their ACC, left hippocampus, and right hippocampus MRS scans, respectively. These participants were, therefore, excluded from their respective analyses (resulting in $N=20,20,18$, and 19 for resting state, ACC, left hippocampus, and right hippocampus scans, respectively).

Spatial preprocessing of functional images was performed in SPM12 and included realignment (motion correction), co-registration of the second BOLD scan with the first BOLD scan, and normalization of BOLD scans to an EPI template in MNI space [80] using a 4th degree B-Spline interpolation. Temporal preprocessing was performed using tools from the Cognitive and Affective Neuroscience Lab (http://github.com/canlab) and included simultaneous bandpass filtering $(0.009-0.08 \mathrm{~Hz})$ and nuisance regression. Nuisance parameters consisted of linear trend, the first 5 principal components of voxels containing cerebrospinal fluid and the first 5 principal components of voxels containing white matter signal (both identified using masks derived from segmented, co-registered, and normalized T1-weighted structural images; [81]), 24 motion parameters from realignment (translations and rotations, their derivatives, and squares of all of these; [82]), and motion censoring or "scrubbing" regressors [83] generated from the ART toolbox using outlier detection and intermediate settings (global-signal $z$-value threshold $=5$, subject-motion threshold $=$ $0.9 \mathrm{~mm}$ ). Finally, these data were parcellated by averaging the BOLD signal at each TR across voxels within a 10- $\mathrm{mm}$ sphere around each of the 264 vertices of the Power atlas [84], producing 264 timeseries of nodes. Susceptibility artifacts due to $7 \mathrm{~T}$ magnetic field inhomogeneities at tissue boundaries led to variable degrees of signal-dropout across participants. On average across scans, $59 \%$ of voxels had acceptable signal-to-noise. However, only 89 Power atlas nodes had acceptable signal-to-noise in at least $75 \%$ of within-sphere voxels across all scans, and thus, functional connectivity data were only analyzed for these 89 ROIs (see Fig. S2 for maps of acceptable signal). Increasing the threshold for allowing nodes into analyses did not qualitatively change results (SI). The split-half reliability of functional connectivity of these 89 nodes was high, and head motion after preprocessing was unrelated to SFC and dFC (Figs. S3-S4).

MRS data were visually inspected to detect any spurious artifact or lipid contamination. Afterwards, spectra were preprocessed using an in-house developed software based on FID-A [85] and quantified with LCModel 6.3 [86] modeling the spectrum from 0.5 to $4.2 \mathrm{ppm}$. Basis sets consisted of 20 metabolites generated using custom-built fully localized density matrix simulations [87]. Macromolecules were simulated in LCModel (NSIMUL = 12). Signal of total creatine was used as an internal standard. Spectra showed overall good spectral quality, though spectra from the left and right hippocampi showed higher variability, especially in the 4-3 ppm region (Fig. S5 and Table S2).

\section{Analyses}

Univariate analyses consisted of one-way ANOVAs and two-tailed $t$ tests comparing clinical, cognitive, and neural baseline measures that occurred $\sim 2$ weeks before the first psilocybin session to those measures postpsilocybin therapy (i.e., 1 week and 4 weeks post-treatment). To measure potential non-treatment-related effects of multiple clinical and cognitive tests, in the delayed group, we compared pre-delay measures to the 

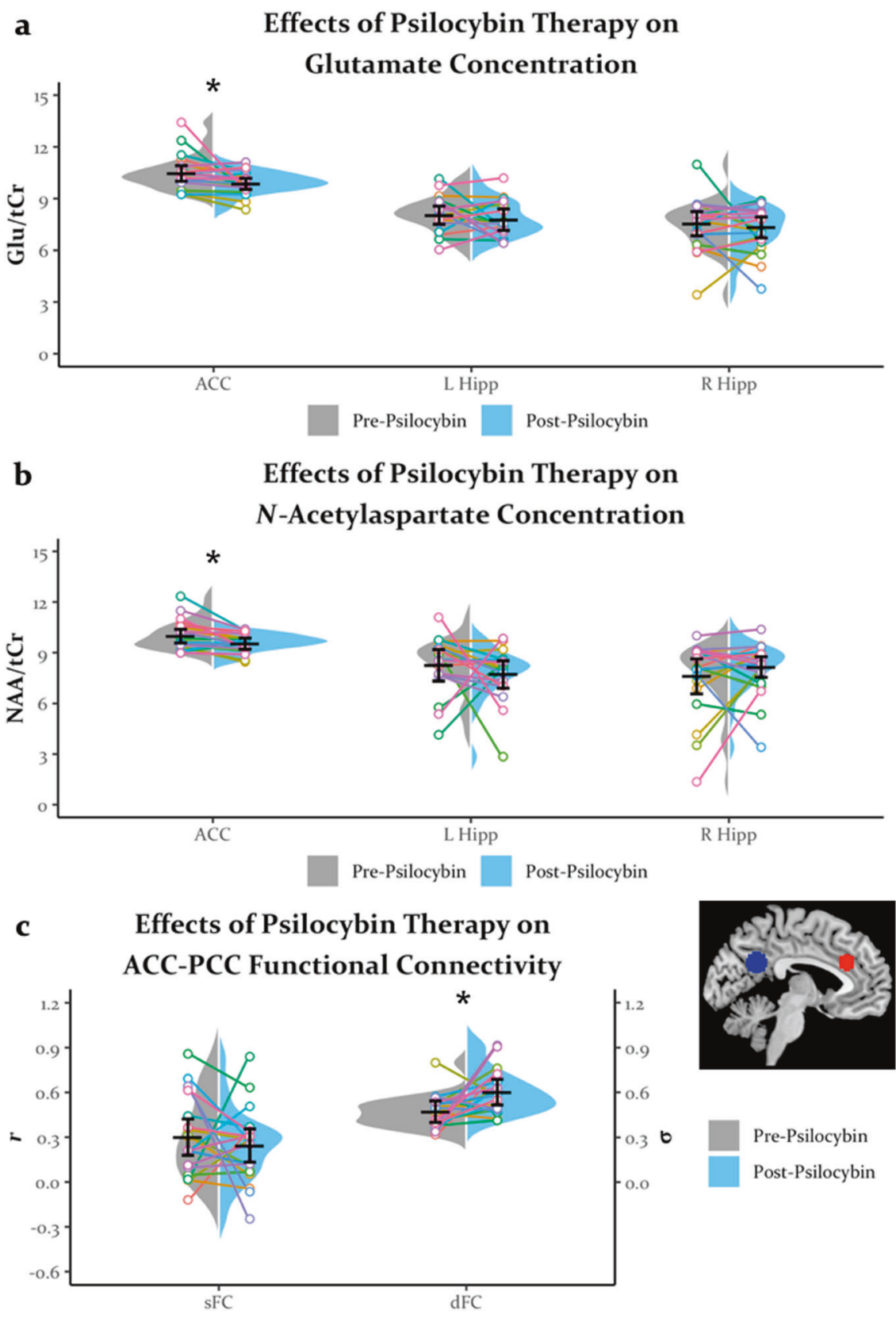

Fig. 2 The effects of psilocybin therapy on neurometabolite concentrations and functional connectivity. Split violin plots with horizontal bars reflecting the mean, error bars reflecting the $95 \%$ confidence interval, and "strings" reflecting individual participant data. The color of a given participant is consistent across all figures plotting individual participant data. Psilocybin therapy reduced (a) glutamate and (b) $N$ acetylaspartate in the anterior cingulate cortex but not in the left or right hippocampus. c Psilocybin therapy increased dFC but not sFC between the anterior and posterior cingulate. ${ }^{*} p<0.050$. ACC anterior cingulate cortex, L Hipp left hippocampus, R Hipp right hippocampus, Glu glutamate, $\mathrm{tCr}$ total creatine, NAA N-acetylaspartate, PCC posterior cingulate.

baseline timepoint 2 weeks before psilocybin therapy and supported null effects with Bayes factors. Exploratory correlations were calculated using Pearson's correlation coefficients.

For analysis of MRS data, we focused on glutamate and NAA, as these are commonly measured metabolites that have reliable spectra, are altered in depression and from antidepressant treatments [74, 75], and have been found to be sensitive to the effects of psychedelics [49]. For the BOLD data, matrices of SFC and dFC were created for each participant and experimental condition (Fig. S6). For SFC, the Pearson's $r$ between the timeseries of all pairwise combinations of 89 nodes was computed, producing 3916 functional connections (edges) with all $r$-values Fisher $z$ transformed for analysis. For $\mathrm{dFC}$, correlation timeseries were first computed for each edge using dynamic conditional correlations (DCC;
[88]), a more reliable method than commonly used sliding window approaches [89]. dFC matrices were then computed by calculating the variance of each correlation timeseries. As described previously, we focused our analyses on the ACC and PCC based on their roles in cognitive flexibility and psychedelic drug action. The specific ACC node was selected based on the Power atlas node contained within the most MRS scans of the ACC. The specific PCC node was selected based on the results of a prior study that found increased SFC between the subgenual cingulate and a region corresponding to the left $\mathrm{PCC} /$ precuneus [53].

In order to test whether baseline measures of $\mathrm{SFC}$ and $\mathrm{dFC}$ predicted post-treatment changes in depression and cognitive flexibility, we constructed and tested the performance of several connectome-based predictive models [90-92]. This approach selects neural features (i.e., 

One-Week Change in ACC-PCC dFC

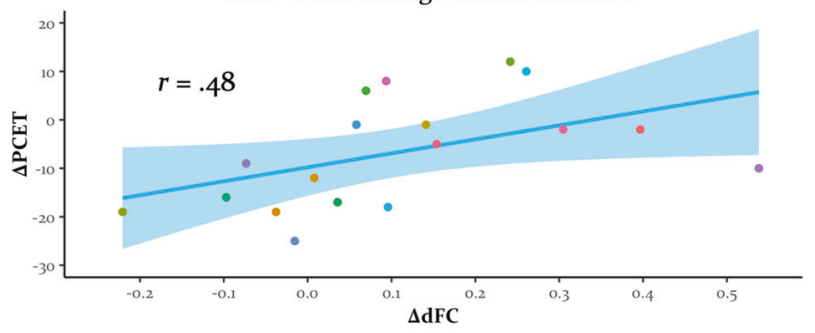

b Four-Week Change in Cognitive Flexibility Against One-Week Change in ACC-PCC dFC

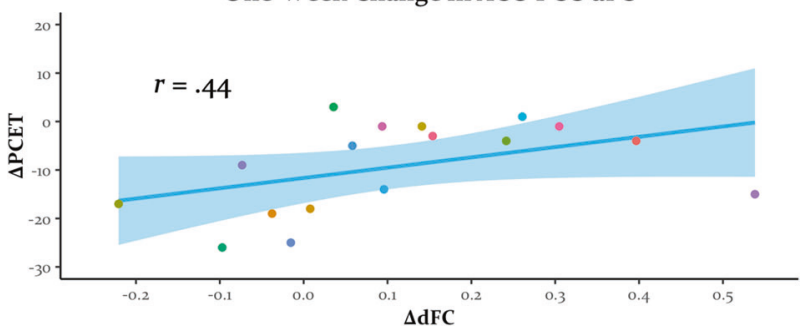

Fig. 3 Greater pre- to post-psilocybin therapy increases in neural flexibility were associated with less improvements in cognitive flexibility. Relationship between changes in anterior to posterior cingulate cortex (ACC and PCC, respectively) dynamics of functional connectivity (dFC) 1 week post-psilocybin therapy and changes in perseverative errors on the Penn Conditional Exclusion Test (PCET) at (a) 1 week and (b) 4 weeks post-psilocybin therapy.

baseline $\mathrm{SFC}$ and $\mathrm{dFC}$ edges) most correlated with behavioral variables (i.e., changes in GRID-HAMD scores and PCET perseverative errors), trains a linear model from a subset of these data by summing these features, and tests these models by applying them to predict behavior from brain data left out of training. We implemented leave-one-participant-out crossvalidation and inspected the relationship between observed and predicted data across folds (Pearson's $r$ ). Features are selected if their correlation with behavior surpasses a thresholded level of significance (typically $p<0.010$ ). Because of the relatively small $N$ and number of edges from which to select, we report the robustness of different models across a range of thresholds for feature selection $(p<0.010-0.050)$.

\section{RESULTS}

Enduring effects of psilocybin therapy on depression and cognitive flexibility

As reported previously [3] and shown in Fig. 1a, psilocybin therapy robustly decreased GRID-HAMD scores in nearly every participant from baseline to 1 week and 4 weeks post-treatment. This reduction in depression was supported by a massive effect of timepoint $\left(F(2,46)=69.95, p<0.001, \eta_{p}^{2}=0.75\right)$. There was moderate evidence supporting the null hypothesis that GRIDHAMD scores did not change between the pre-delay and baseline timepoint in the delayed group before they received psilocybin $(F(2,20)=0.25, p>0.250, \mathrm{BF}=0.23)$.

Similar to GRID-HAMD scores, perseverative errors on the PCET generally decreased from baseline to 1 week post-psilocybin therapy (Fig. 1b) with this effect sustained 4 weeks post-treatment. This improvement in cognitive flexibility was supported by a main effect of timepoint $\left(F(2,40)=10.90, p<0.001, \eta_{P}^{2}=0.35\right)$. The effect of psilocybin therapy on PCET perseverative errors was unlikely to be explained by practice effects, as there was moderate evidence supporting the null hypothesis that perseverative errors did not change in the delayed group prior to receiving psilocybin $(95 \% \mathrm{Cl}=$ $[-9.77,10.57], t(9)=0.09, p>0.250, \mathrm{BF}=0.31)$. Changes in GRIDHAMD scores were not correlated with changes in PCET perseverative errors across any set of timepoints (all $|r| s<0.2$, all $p s>0.250$ ).
Enduring effects of Psilocybin therapy on brain function

Both glutamate $(95 \% \mathrm{Cl}=[0.11,1.10], t(19)=2.54, p=0.020, d=$ $0.57)$ and NAA $(t(19)=3.05, p=0.007, d=0.68)$ were decreased in the ACC 1 week after psilocybin therapy (Fig. 2a, b). Decreases in ACC neurometabolite concentrations were regionally selective, as there were no significant changes in glutamate and NAA in the left or right hippocampi (all $t s<1.04$, all $p s>0.250$ ). All correlations between changes in glutamate or NAA and changes in GRIDHAMD scores or PCET perseverative errors were non-significant (all $|r| s<.36$, all $p s>0.130)$.

In general, sFC numerically decreased and dFC numerically increased across the brain after psilocybin therapy (Fig. S7). Prior work found increases in SFC between the ACC and PCC 1 day after ayahuasca administration [51]. Therefore, we examined the effects of psilocybin therapy on ACC-PCC functional connectivity. Although we observed no significant change in $\mathrm{sFC}(95 \% \mathrm{Cl}=[-0.12,0.23]$; $t$ $(19)=0.67, p>0.250)$, there was a fairly reliable increase in dFC between the ACC and PCC 1 week after psilocybin therapy $(95 \% \mathrm{Cl}$ $=[0.02,0.21] ; t(19)=2.87, p=0.010, d=0.64$; Fig. $2 \mathrm{c}$ ). Exploratory correlations found a moderate association between these increases in $\mathrm{dFC}$ and decreases in PCET perseverative errors 1 week postpsilocybin therapy $(r(16)=0.48, p=0.043$; Fig. 3a) with a slightly smaller non-significant correlation observed at 4 weeks postpsilocybin therapy $(r(16)=0.44, p=0.079$; Fig. 3b). Interestingly, these correlations were positive, suggesting that an increase in dFC may actually be associated with less of a decrease in perseverative errors (i.e., less increase in cognitive flexibility). All changes in ACCPCC functional connectivity and changes in GRID-HAMD scores were non-significant (all $|r| s<0.10$, all $p s>0.250$ ).

\section{Prediction of improvement from baseline functional connectivity}

A goal of clinical neuroimaging is to use tools from cognitive neuroscience to identify biomarkers to inform the diagnosis and treatment of psychiatric disorders. In order to explore such possibilities and further elucidate the counterintuitive findings that greater psilocybin-induced neural changes can be associated with less improvement in cognitive flexibility, we trained connectome-based predictive models on baseline functional connectivity to predict changes in depression and cognitive flexibility at 1 and 4 weeks post-psilocybin therapy. Models for each behavioral variable were trained on baseline SFC, $\mathrm{dFC}$, and both SFC and AFC of the ACC, PCC, both ACC and PCC, and the connectome of 3916 edges (the "full" connectome). Performance of these models across a range of thresholds for feature selection is shown in Fig. 4.

There are several points worth noting regarding these predictive models. No model was capable of predicting 1-week improvements in depression (Fig. 4a). In contrast, the prediction of 4-week improvements in depression was moderately good and consistent across thresholds for feature selection using the model trained on baseline SFC of the full connectomre (Fig. 4b). Successful prediction of 1-week and 4-week changes in cognitive flexibility was best with both baseline SFC and dFC of the ACC with the addition of edges emanating from the PCC reducing model performance (Fig. 4c, d), though both models contained edges from the ACC to posteromedial regions. These models predicting changes in cognitive flexibility were fairly robust to relaxing the threshold for edge selection.

To further interrogate predictive model features, we examined the relationship between individual model features (sFC and dFC edges) and behavioral variables across thresholds for feature selection. Whereas baseline sFC and dFC edges predicting 4-week improvements in depression could be both positively and negatively correlated with changes in depression (Fig. S8), the best models predicting 1 -week and 4-week changes in cognitive flexibility (i.e., sFC and $\mathrm{dFC}$ of the ACC) contained features that were counterintuitively related to behavior. Specifically, baseline 

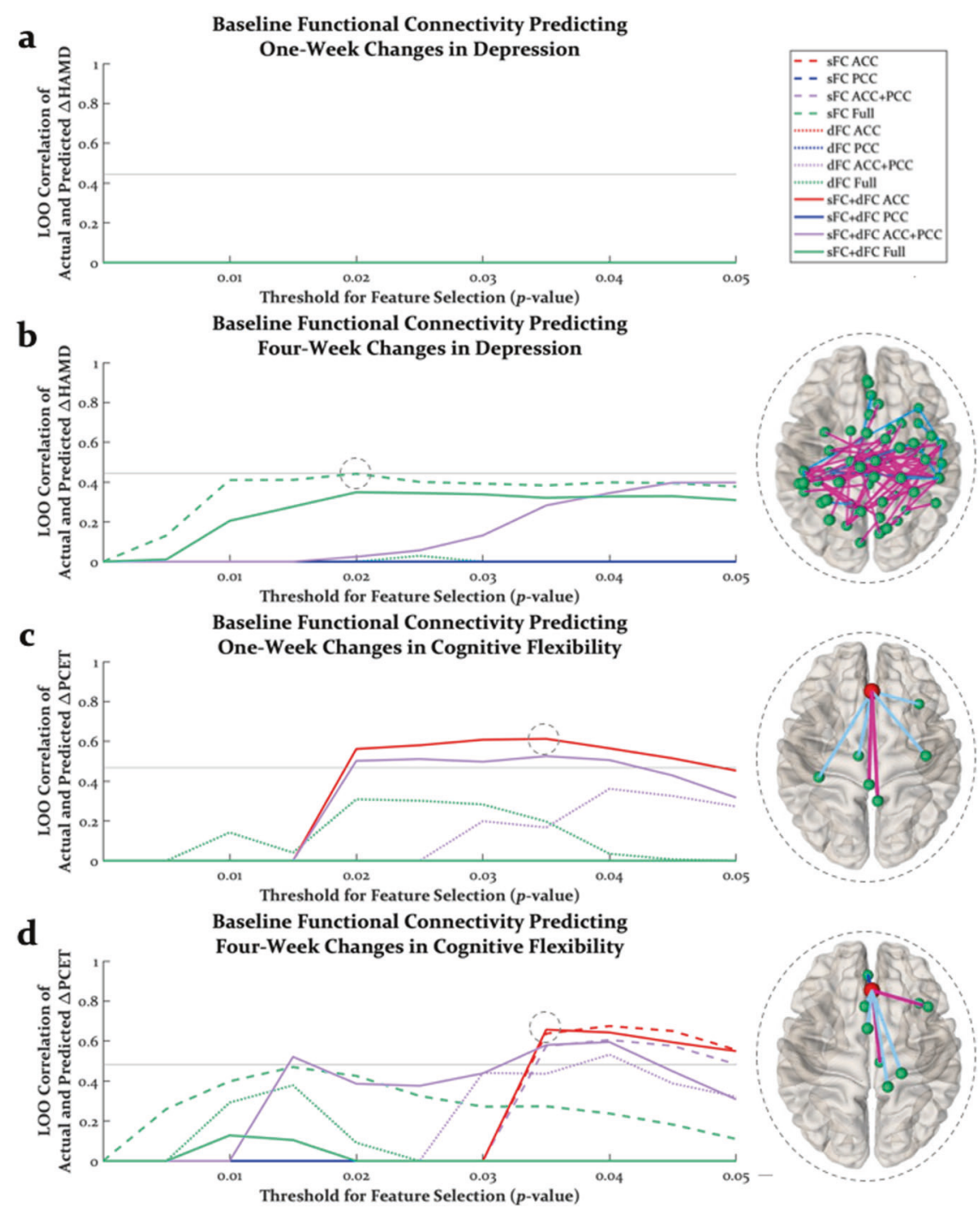

Fig. 4 Connectome-based predictive modeling of pre- to post-psilocybin therapy changes in depression and cognitive flexibility. Performance of models trained on baseline functional connectivity as a function of the threshold for feature selection predicting 1-week (a) and 4-week (b) changes in depression $(\triangle \mathrm{HAMD})$ and 1-week (c) and 4-week (d) changes in cognitive flexibility $(\triangle \mathrm{PCET})$. Horizontal gray lines indicate model performance at $p=0.05$. Brains on the right side of panels are the best-performing models (see gray circles on performance lines). A brain was not plotted for predicting 1-week changes in depression, as there was no model was that performed at $r>0$. Edges that were included in at least $75 \%$ of folds were plotted in dark blue, magenta, and light blue, representing sFC edges positively correlated with behavior, sFC edges negatively correlated behavior, and dFC edges positively correlated with behavior, respectively. Brain visualizations created with NeuroMArVL (https://immersive.erc.monash.edu/neuromarvl/). LOO leave-one-out, sFC static functional connectivity, dFC dynamics of functional connectivity, ACC anterior cingulate cortex, PCC posterior cingulate cortex.

$\mathrm{dFC}$ edges were selected more often than SFC edges, and these $\mathrm{dFC}$ edges were positively correlated with the reductions in PCET perseverative errors (Fig. $5 \mathrm{a}, \mathrm{b}$ ). In contrast, when models were trained on baseline functional connectivity to predict baseline cognitive flexibility (Fig. 5c), again more dFC edges were selected than SFC edges, but these brain-behavior correlations were strikingly reversed (Fig. $5 \mathrm{~d}$ ). That is, at baseline, greater $\mathrm{dFC}$ was predictive of greater cognitive flexibility (less PCET perseverative errors), but after psilocybin therapy, which tended to increase brain-wide dFC, greater baseline dFC was associated with less improvement in cognitive flexibility. Similar to models predicting changes in depression, models trained on baseline functional connectivity to predict baseline depression involved sFC and dFC that were both positively and negatively correlated with depression (Fig. S8). Finally, training predictive models only on nodes with acceptable signal-to-noise in $100 \%$ of within-sphere voxels across all scans produced qualitatively similar results that did not change the interpretation of these results (Figs. S9-S11).

\section{DISCUSSION}

Psilocybin therapy was shown to increase cognitive and neural flexibility in patients with MDD. Increases in cognitive flexibility were selective, as tasks measuring response inhibition, selective attention, and abstract reasoning were not impacted. Although a lack of correlation between improvements in cognitive flexibility and improvements in depression might suggest that cognitive flexibility is not mechanistically related to antidepressant effects of psychedelic therapy, the GRID-HAMD assesses different features of current depression (e.g., affect and appetite) and not necessarily cognitive impairments that may pre-date treatment seeking [13] or that persist in those who otherwise respond to treatment 
a

Number of Baseline Edges Selected in $\geq 75 \%$ of Folds of

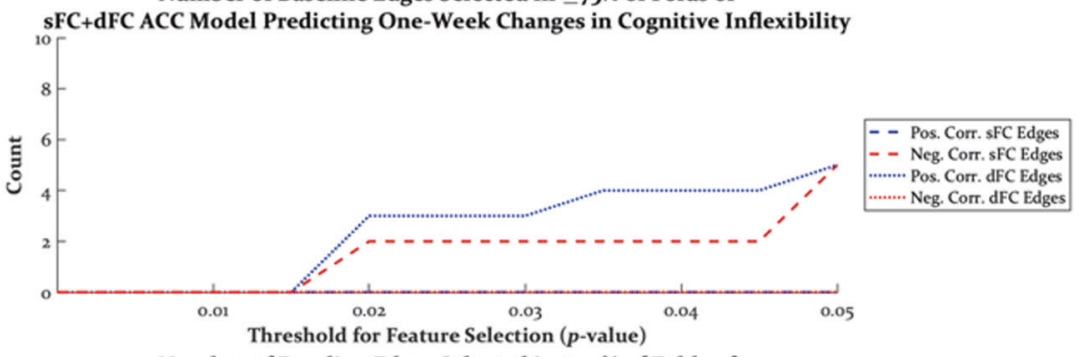

b

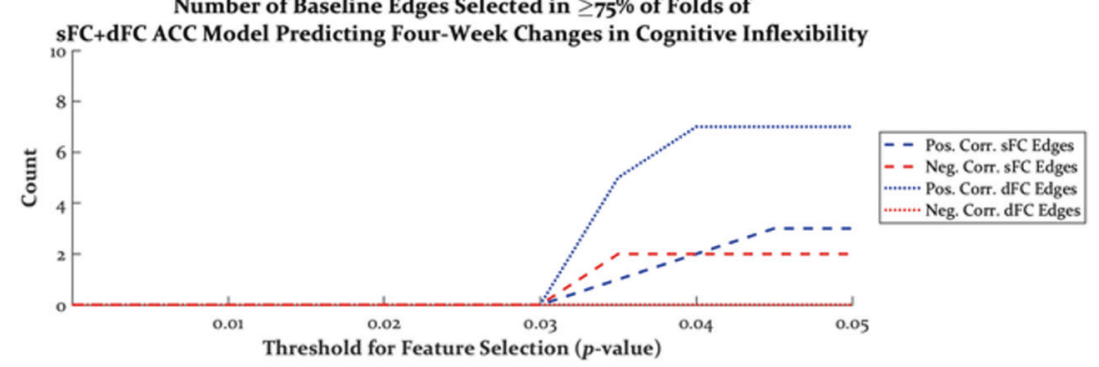

c

Baseline Functional Connectivity Predicting

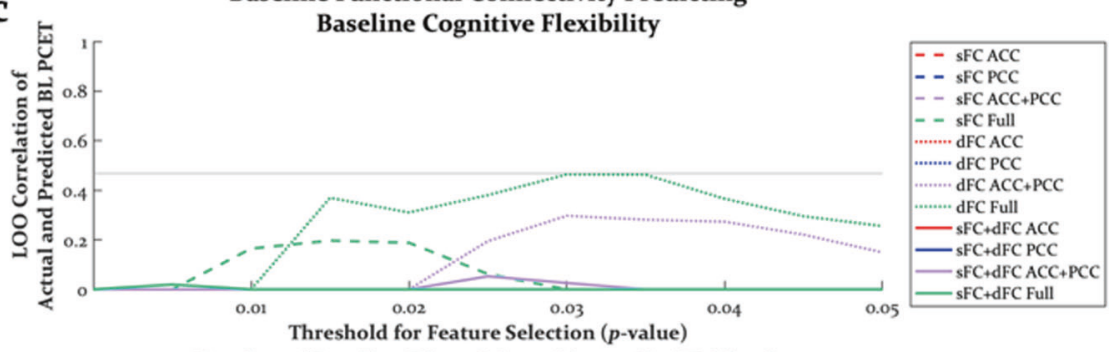

d

Number of Baseline Edges Selected in $\geq 75 \%$ of Folds of

SFC+dFC Full Model Predicting Baseline Cognitive Inflexibility

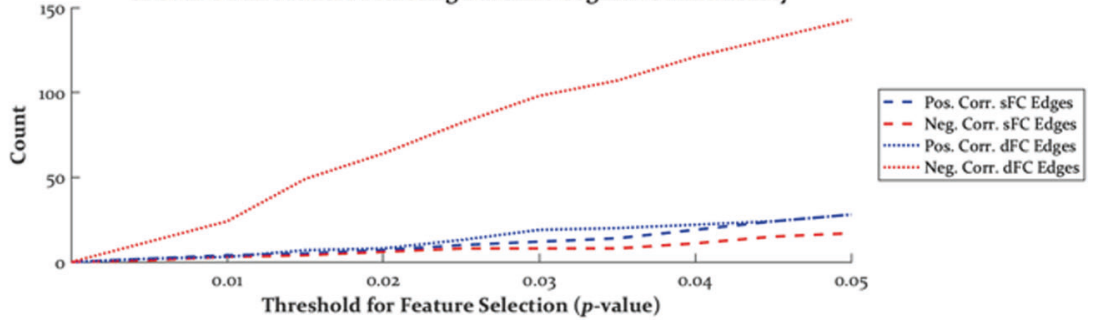

Fig. 5 The relationship between model features and cognitive flexibility. Positively and negatively correlated features that were selected in the best models trained on baseline SFC and dFC predicting (a) 1-week changes and (b) 4-week changes in cognitive flexibility. Positively correlated dFC edges were consistently selected in these models, suggesting that greater baseline $\mathrm{dFC}$ was associated with more PCET perseverative errors (i.e., greater cognitive rigidity). In contrast, when models were trained on baseline dFC to predict baseline cognitive flexibility (c), dFC edges were still predictive of cognitive flexibility, but the correlations between $\mathrm{dFC}$ edges and cognitive flexibility was reversed (d). The SFC + dFC Full model was plotted here to highlight that regardless of how many edges were allowed into the model, far more dFC edges were negatively correlated with PCET perseverative errors. Pos. positively, Neg. negatively, Corr. correlated, LOO leave-oneout, SFC static functional connectivity, dFC dynamics of functional connectivity, ACC anterior cingulate cortex, PCC posterior cingulate cortex.

$[28,29]$. While improved cognitive flexibility itself is a noteworthy and important effect of psilocybin therapy, it is possible that enhancements in cognitive and neural flexibility may open a window of plasticity [56] during which improvements can be facilitated (e.g., with supportive psychotherapy), though this remains speculative. In the current sample, despite psilocybin therapy generally increasing dFC across the brain, the benefits of this neural flexibility were nonlinear. That is, larger pre- to posttreatment increases in dFC between the ACC and PCC and greater baseline dFC of the ACC was associated with less improvement in cognitive flexibility. An implication of this finding is that subpopulations of patients (i.e., those with lower baseline neural flexibility) may be more likely to benefit from psychedelic therapy.

This study had several limitations. The study design was not placebo-controlled and instead utilized a pre- vs. post-treatment design, suggesting that the observed effects could be attributable to expectancy, practice, or exposure effects. We believe this is an insufficient explanation, as the effects of psilocybin therapy on depression were far larger than typical placebo effects [93, 94], and there was no evidence for practice effects on the PCET in the delayed group that performed the task twice before receiving psilocybin therapy. Moreover, it seems implausible for certain biological signals to be decreased by placebo effects such as ACC glutamate, which has good test-retest reliability [95]. Nevertheless, in a recent double-blind clinical trial on the treatment of MDD comparing psilocybin to the SSRI citalopram using equivalent protocols for psychotherapy in both conditions [7], citalopram was found to be effective, though somewhat less so than psilocybin, 1 week after treatment, much sooner than is typically expected with a SSRI. Another study found that a trivial dose of psilocybin in 
the context of the psychotherapy that accompanies psilocybin therapy had considerable reductions on depression [4]. Thus, one could reasonably interpret that large doses of psychotherapy and expectancy of receiving psilocybin provide for a substantial therapeutic element of psilocybin therapy. Another limitation to this study was that due to signal dropout at $7 \mathrm{~T}$, we unfortunately lost a large portion of brain regions, though including more or less noisy regions into analyses did not qualitatively change our results. In addition, the relatively few edges of the ACC-based predictive model of cognitive flexibility might actually speak to its robustness because there were likely missing edges that include other regions known to support cognitive flexibility (e.g., orbitofrontal cortex; [8]). However, signal dropout in various regions implicated in the pathophysiology of mood disorders (e.g., the hippocampus [26]) may have precluded finding betterperforming models to predict changes in depression after psilocybin therapy. Considering the heterogeneity of depression [96], such better-predicting models may rely on a more distributed network of brain regions than we were able to sample within our data. Finally, the generalizability of predictive models is expected to vary as a function of sample size. The current findings are based on a relatively small sample size for predictive models, though a slightly larger sample $(N=25)$ was previously used to build a predictive model of attention that has consistently generalized to other measures of attention, clinical symptoms, and acute drug effects [97-99].

Our findings support a role of the ACC, as well as other parts of the cingulate gyrus, in the enduring effects of psilocybin on cognition. Consistent with past work that found changes in interactions between the ACC and PCC [51] and the subgenual cingulate and PCC [53] 1 day after psychedelic administration, we found increased dFC between the ACC and PCC. Moreover, models trained only on ACC functional connectivity to predict cognitive flexibility outperformed all other models, including those that could select features from the full connectome, which had over 40 times the number of edges than a single node. Finally, the selective decreases in both glutamate and NAA of the ACC suggest a reduction in neural metabolism, though further work will be needed to determine how such reductions in metabolism relate to increased neural flexibility.

The possibility that greater neural flexibility might attenuate the benefits of psilocybin therapy to cognitive flexibility should perhaps not be surprising. Patients with schizophrenia, for example, exhibit increased brain wide neural flexibility [67]. It could be that psilocybin pushes neural flexibility in some individuals past the zone of largest therapeutic efficacy. One question that our study was not designed to address is whether such detrimental effects are due to too much neural flexibility or too enduring of elevations in neural flexibility. Neural flexibility could be required during the acute effects of psychedelics to permit the exploration of novel cognitive states that can allow one to escape maladaptive attractor basins (e.g., rumination). Perhaps even in the days following the acute effects, some neural flexibility may be needed for the integration process, allowing for greater responsiveness to continuing psychotherapy. However, persisting increases in neural flexibility could become destabilizing to an individual's life, resulting in, for example, lower attention [90, 100]. It may be that psychedelics with longer or shorter enduring effects are more beneficial for different psychiatric disorders. Overall, our work suggests that psilocybin therapy may improve cognitive flexibility in psychiatric illness, but it highlights potential boundary conditions of psychedelic-induced neural flexibility and its relationship to cognitive improvements.

\section{CODE AVAILABILITY}

Computed code used to analyze data or generate figures can be requested from MKD.

\section{REFERENCES}

1. Bogenschutz MP, Forcehimes AA, Pommy JA, Wilcox CE, Barbosa P, Strassman RJ. Psilocybin-assisted treatment for alcohol dependence: a proof-of-concept study. J Psychopharmacol. 2015;29:289-99.

2. Carhart-Harris RL, Bolstridge M, Rucker J, Day CMJ, Erritzoe D, Kaelen M, et al. Psilocybin with psychological support for treatment-resistant depression: an open-label feasibility study. Lancet Psychiatry. 2016;3:619-27.

3. Davis AK, Barrett FS, May DG, Cosimano MP, Sepeda ND, Johnson MW, et al. Effects of psilocybin-assisted therapy on major depressive disorder: a randomized clinical trial. JAMA Psychiatry. 2020. https://doi.org/10.1001/ jamapsychiatry.2020.3285.

4. Griffiths RR, Johnson MW, Carducci MA, Umbricht A, Richards WA, Richards BD, et al. Psilocybin produces substantial and sustained decreases in depression and anxiety in patients with life-threatening cancer: a randomized double-blind trial. J Psychopharmacol. 2016;30:1181-97.

5. Johnson MW, Garcia-Romeu A, Cosimano MP, Griffiths RR. Pilot study of the 5-HT ${ }_{2 \mathrm{~A}} \mathrm{R}$ agonist psilocybin in the treatment of tobacco addiction. J Psychopharmacol. 2014;28:983-92.

6. Moreno FA, Wiegand CB, Taitano EK, Delgado PL. Safety, tolerability, and efficacy of psilocybin in 9 patients with obsessive-compulsive disorder. J Clin Psychiatry. 2006;67:1735-40.

7. Carhart-Harris R, Giribaldi B, Watts R, Baker-Jones M, Murphy-Beiner A, Murphy $\mathrm{R}$, et al. Trial of psilocybin versus escitalopram for depression. N. Engl J Med. 2021;384:1402-11.

8. Uddin LQ Cognitive and behavioural flexibility: neural mechanisms and clinical considerations. Nat Rev Neurosci. 2021. https://doi.org/10.1038/s41583-02100428-w.

9. Stange JP, Alloy LB, Fresco DM. Inflexibility as a vulnerability to depression: a systematic qualitative review. Clin Psychol: Sci Pract. 2017;24:245-76.

10. Channon S. Executive dysfunction in depression: The Wisconsin Card Sorting Test. J Affect Disord. 1996;39:107-14.

11. Gruner $P$, Pittenger C. Cognitive inflexibility in obsessive-compulsive disorder. Neuroscience. 2017;345:243-55.

12. Verdejo-Garcia A, Clark L, Verdejo-Román J, Albein-Urios N, Martinez-Gonzalez JM, Gutierrez B, et al. Neural substrates of cognitive flexibility in cocaine and gambling addictions. Br J Psychiatry. 2015;207:158-64.

13. Maramis MM, Mahajudin MS, Khotib J. Impaired cognitive flexibility and working memory precedes depression: a rat model to study depression. Neuropsychobiology. 2021;80:225-33.

14. Dotson VM, McClintock SM, Verhaeghen P, Kim JU, Draheim AA, Syzmkowicz $\mathrm{SM}$, et al. Depression and cognitive control across the lifespan: a systematic review and meta-analysis. Neuropsychol Rev. 2020;30:461-76.

15. Goodall J, Fisher C, Hetrick S, Phillips L, Parrish EM, Allott K. Neurocognitive functioning in depressed young people: a systematic review and meta-analysis. Neuropsychol Rev. 2018;28:216-31.

16. Liu H, Funkhouser $C J$, Langenecker SA, Shankman SA. Set shifting and inhibition deficits as potential endophenotypes for depression. Psychiatry Res. 2021;300:113931.

17. Greer TL, Sunderajan P, Grannemann BD, Kurian BT, Trivedi MH. Does duloxetine improve cognitive function independently of its antidepressant effect in patients with major depressive disorder and subjective reports of cognitive dysfunction? Depression Res Treat. 2014;2014:1-13.

18. Johnco C, Wuthrich VM, Rapee RM. The influence of cognitive flexibility on treatment outcome and cognitive restructuring skill acquisition during cognitive behavioural treatment for anxiety and depression in older adults: results of a pilot study. Behav Res Ther. 2014;57:55-64.

19. Davis AK, Barrett FS, Griffiths RR. Psychological flexibility mediates the relations between acute psychedelic effects and subjective decreases in depression and anxiety. J Contextual Behav Sci. 2020;15:39-45.

20. Shiroma PR, Thuras P, Wels J, Albott CS, Erbes C, Tye S, et al. Neurocognitive performance of repeated versus single intravenous subanesthetic ketamine in treatment resistant depression. J Affect Disord. 2020;277:470-7.

21. Nielen MMA, Den Boer JA. Neuropsychological performance of OCD patients before and after treatment with fluoxetine: evidence for persistent cognitive deficits. Psychol Med. 2003;33:917-25.

22. Boggio PS, Fregni F, Bermpohl F, Mansur CG, Rosa M, Rumi DO, et al. Effect of repetitive TMS and fluoxetine on cognitive function in patients with Parkinson's disease and concurrent depression. Mov Disord. 2005;20:1178-84.

23. Baños JH, Novack TA, Brunner R, Renfroe S, Lin H-Y, Meythaler J. Impact of early administration of sertraline on cognitive and behavioral recovery in the first year after moderate to severe traumatic brain injury. J Head Trauma Rehabilitation. 2010;25:357-61.

24. Jett JD, Boley AM, Girotti M, Shah A, Lodge DJ, Morilak DA. Antidepressant-like cognitive and behavioral effects of acute ketamine administration associated 
with plasticity in the ventral hippocampus to medial prefrontal cortex pathway. Psychopharmacology. 2015;232:3123-33.

25. Marwari S, Dawe GS. (R)-fluoxetine enhances cognitive flexibility and hippocampal cell proliferation in mice. J Psychopharmacol. 2018;32:441-57.

26. MacQueen G, Frodl T. The hippocampus in major depression: evidence for the convergence of the bench and bedside in psychiatric research? Mol Psychiatry. 2011;16:252-64.

27. Park AJ, Harris AZ, Martyniuk KM, Chang C-Y, Abbas Al, Lowes DC, et al. Reset of hippocampal-prefrontal circuitry facilitates learning. Nature. 2021;591:615-9.

28. Snyder HR. Major depressive disorder is associated with broad impairments on neuropsychological measures of executive function: a meta-analysis and review. Psychological Bull. 2013;139:81-132.

29. Rock PL, Roiser JP, Riedel WJ, Blackwell AD. Cognitive impairment in depression: a systematic review and meta-analysis. Psychol Med. 2014;44:2029-40.

30. Boulougouris V, Glennon JC, Robbins TW. Dissociable effects of selective 5-HT2A and $5-\mathrm{HT} 2 \mathrm{C}$ receptor antagonists on serial spatial reversal learning in rats. Neuropsychopharmacol. 2008;33:2007-19.

31. Furr A, Lapiz-Bluhm MD, Morilak DA. 5-HT2A receptors in the orbitofrontal cortex facilitate reversal learning and contribute to the beneficial cognitive effects of chronic citalopram treatment in rats. Int J Neuropsychopharm. 2012;15:1295-305.

32. Amodeo DA, Rivera E, Cook EH, Sweeney JA, Ragozzino ME. 5HT 2 A receptor blockade in dorsomedial striatum reduces repetitive behaviors in BTBR mice: striatal $5 \mathrm{HT}_{2 \mathrm{~A}}$ blockade improves repetitive behaviors. Genes, Brain Behav. 2017; 16:342-51.

33. Amodeo DA, Jones JH, Sweeney JA, Ragozzino ME. Risperidone and the 5-HT $2 \mathrm{~A}$ receptor antagonist M100907 improve probabilistic reversal learning in BTBR T $+\mathrm{tf} / \mathrm{J}$ mice: $5 \mathrm{HT}_{2 \mathrm{~A}}$ receptor blockade improves reversal learning in BTBR mice. Autism Res. 2014;7:555-67.

34. Baker PM, Thompson JL, Sweeney JA, Ragozzino ME. Differential effects of 5-HT2A and 5-HT2C receptor blockade on strategy-switching. Behavioural Brain Res. 2011;219:123-31.

35. Pokorny T, Duerler P, Seifritz E, Vollenweider FX, Preller KH. LSD acutely impairs working memory executive functions and cognitive flexibility but not risk-based decision-making. Psychol Med. 2020;50:2255-64. https://doi.org/10.1017/ S0033291719002393.

36. Amodeo DA, Hassan O, Klein L, Halberstadt AL, Powell SB. Acute serotonin 2A receptor activation impairs behavioral flexibility in mice. Behavioural Brain Res. 2020;395:112861.

37. Odland AU, Kristensen JL, Andreasen JT. The selective 5-HT2A receptor agonist $25 \mathrm{CN}-\mathrm{NBOH}$ does not affect reversal learning in mice. Behav Pharmacol. 2021. https://doi.org/10.1097/FBP.0000000000000626.

38. Boulougouris V, Robbins TW. Enhancement of spatial reversal learning by $5-\mathrm{HT} 2 \mathrm{C}$ receptor antagonism is neuroanatomically specific. J Neurosci. 2010;30:930-8

39. Dajani DR, Odriozola P, Winters M, Voorhies W, Marcano S, Baez A, et al. Measuring cognitive flexibility with the flexible item selection task: from fMRI adaptation to individual connectome mapping. J Cogn Neurosci. 2020:32:1026-45.

40. Ghahremani DG, Monterosso J, Jentsch JD, Bilder RM, Poldrack RA. Neural components underlying behavioral flexibility in human reversal learning. Cereb Cortex. 2010;20:1843-52

41. Cohen Y, Schneidman E, Paz R. The geometry of neuronal representations during rule learning reveals complementary roles of cingulate cortex and putamen. Neuron. 2021: S089662732031031X

42. Vollenweider F. Positron emission tomography and fluorodeoxyglucose studies of metabolic hyperfrontality and psychopathology in the psilocybin model of psychosis. Neuropsychopharmacology. 1997;16:357-72.

43. Gouzoulis-Mayfrank E. Neurometabolic effects of psilocybin, 3,4-methylenedioxyethylamphetamine (MDE) and d-methamphetamine in healthy volunteers A double-blind, placebo-controlled PET study with [18F]FDG. Neuropsychopharmacology. 1999;20:565-81.

44. Riba J, Romero S, Grasa E, Mena E, Carrió I, Barbanoj MJ. Increased frontal and paralimbic activation following ayahuasca, the pan-amazonian inebriant. Psychopharmacology. 2006;186:93-98.

45. Carhart-Harris RL, Leech R, Erritzoe D, Williams TM, Stone JM, Evans J, et al Functional connectivity measures after psilocybin inform a novel hypothesis of early psychosis. Schizophrenia Bull. 2013;39:1343-51.

46. Carhart-Harris RL, Muthukumaraswamy S, Roseman L, Kaelen M, Droog W, Murphy K, et al. Neural correlates of the LSD experience revealed by multimoda neuroimaging. Proc Natl Acad Sci USA. 2016;113:4853-8.

47. Müller F, Dolder PC, Schmidt A, Liechti ME, Borgwardt S. Altered network hub connectivity after acute LSD administration. Neurolmage: Clin 2018;18:694-701.
48. Pasquini L, Palhano-Fontes F, Araujo DB. Subacute effects of the psychedelic ayahuasca on the salience and default mode networks. J Psychopharmacol. 2020;34:623-35.

49. Sampedro $F$, de la Fuente Revenga $M$, Valle $M$, Roberto $N$, Domínguez-Clavé $E$, Elices $M$, et al. Assessing the psychedelic "after-glow" in Ayahuasca users: postacute neurometabolic and functional connectivity changes are associated with enhanced mindfulness capacities. Int J Neuropsychopharmacol. 2017;20:698-711.

50. Carhart-Harris RL, Erritzoe D, Williams T, Stone JM, Reed $\amalg$, Colasanti A, et al Neural correlates of the psychedelic state as determined by fMRI studies with psilocybin. Proc Natl Acad Sci USA. 2012;109:2138-43.

51. Palhano-Fontes F, Andrade KC, Tofoli LF, Santos AC, Crippa JAS, Hallak JEC, et al. The psychedelic state induced by Ayahuasca modulates the activity and connectivity of the default mode network. PLOS ONE. 2015;10:e0118143.

52. Preller KH, Razi A, Zeidman $P$, Stämpfli $P$, Friston KJ, Vollenweider FX. Effective connectivity changes in LSD-induced altered states of consciousness in humans. Proc Natl Acad Sci USA. 2019;116:2743-8.

53. Carhart-Harris RL, Roseman L, Bolstridge $M$, Demetriou $L$, Pannekoek JN, Wall $M B$, et al. Psilocybin for treatment-resistant depression: $\mathrm{fMRI}-$ measured brain mechanisms. Sci Rep. 2017;7. https://doi.org/10.1038/s41598-017-13282-7.

54. Hamani C, Mayberg H, Stone S, Laxton A, Haber S, Lozano AM. The subcallosal cingulate gyrus in the context of major depression. Biol Psychiatry. 2011;69:301-8.

55. Schwartz J, Ordaz SJ, Kircanski K, Ho TC, Davis EG, Camacho MC, et al. Restingstate functional connectivity and inflexibility of daily emotions in major depression. J Affect Disord. 2019;249:26-34.

56. Ly C, Greb AC, Cameron LP, Wong JM, Barragan EV, Wilson PC, et al. Psychedelics promote structural and functional neural plasticity. Cell Rep. 2018;23:3170-82.

57. Nardou R, Lewis EM, Rothhaas R, Xu R, Yang A, Boyden E, et al. Oxytocindependent reopening of a social reward learning critical period with MDMA. Nature. 2019:569:116-20.

58. Morales-Garcia JA, Calleja-Conde J, Lopez-Moreno JA, Alonso-Gil S, SanzSanCristobal M, Riba J, et al. N,N-dimethyltryptamine compound found in the hallucinogenic tea ayahuasca, regulates adult neurogenesis in vitro and in vivo. Transl Psychiatry. 2020;10:331.

59. Catlow BJ, Song S, Paredes DA, Kirstein CL, Sanchez-Ramos J. Effects of psilocybin on hippocampal neurogenesis and extinction of trace fear conditioning. Exp Brain Res. 2013;228:481-91.

60. Cameron LP, Tombari RJ, Lu J, Pell AJ, Hurley ZQ, Ehinger $\mathrm{Y}$, et al. A nonhallucinogenic psychedelic analogue with therapeutic potential. Nature. 2021;589:474-9.

61. Inserra A, De Gregorio D, Gobbi G. Psychedelics in psychiatry: neuroplastic, immunomodulatory, and neurotransmitter mechanisms. Pharm Rev. 2021;73:202-77.

62. Olson DE. sychoplastogens: A promising class of plasticity-promoting neurotherapeutics. J Exp Neurosci. 2018;12:117906951880050.

63. Chen T, Cai W, Ryali S, Supekar K, Menon V. Distinct global brain dynamics and spatiotemporal organization of the salience network. PLoS Biol. 2016;14: e1002469.

64. Waschke L, Kloosterman NA, Obleser J, Garrett DD. Behavior needs neural variability. Neuron. 2021: S0896627321000453.

65. Zheng H, Li F, Bo Q, Li X, Yao L, Yao Z, et al. The dynamic characteristics of the anterior cingulate cortex in resting-state $\mathrm{fMRI}$ of patients with depression. J Affect Disord. 2018;227:391-7.

66. Lin $C$, Lee $S-H$, Huang $C-M$, Chen $G-Y$, Ho P-S, Liu H-L, et al. Increased brain entropy of resting-state $\mathrm{fMRI}$ mediates the relationship between depression severity and mental health-related quality of life in late-life depressed elderly. J Affect Disord. 2019;250:270-7.

67. Braun U, Schäfer A, Bassett DS, Rausch F, Schweiger Jl, Bilek E, et al. Dynamic brain network reconfiguration as a potential schizophrenia genetic risk mechanism modulated by NMDA receptor function. Proc Natl Acad Sci USA. 2016;113:12568-73.

68. Carhart-Harris RL, Leech R, Hellyer PJ, Shanahan M, Feilding A, Tagliazucchi E, et al. The entropic brain: a theory of conscious states informed by neuroimaging research with psychedelic drugs. Front Human Neurosci. 2014;8. https://doi.org/ 10.3389/fnhum.2014.00020.

69. Lebedev AV, Kaelen M, Lövdén M, Nilsson J, Feilding A, Nutt DJ, et al. LSDinduced entropic brain activity predicts subsequent personality change: LSDinduced entropic brain activity. Hum Brain Mapp. 2016;37:3203-13.

70. Tagliazucchi E, Carhart-Harris R, Leech R, Nutt D, Chialvo DR. Enhanced repertoire of brain dynamical states during the psychedelic experience: Enhanced Repertoire of Brain Dynamical States. Hum Brain Mapp. 2014;35:5442-56.

71. Doss MK, May DG, Johnson MW, Clifton JM, Hedrick SL, Prisinzano TE, et al. The acute effects of the atypical dissociative hallucinogen salvinorin $A$ on functional 
connectivity in the human brain. Sci Rep. 2020;10. https://doi.org/10.1038/ s41598-020-73216-8.

72. Spies M, Klöbl M, Höflich A, Hummer A, Vanicek T, Michenthaler P, et al. Association between dynamic resting-state functional connectivity and ketamine plasma levels in visual processing networks. Sci Rep. 2019;9. https://doi.org/ 10.1038/s41598-019-46702-x.

73. Barrett FS, Doss MK, Sepeda ND, Pekar JJ, Griffiths RR. Emotions and brain function are altered up to one month after a single high dose of psilocybin. Sci Rep. 2020;10. https://doi.org/10.1038/s41598-020-59282-y.

74. Abdallah CG, Hannestad J, Mason GF, Holmes SE, DellaGioia N, Sanacora G, et al. Metabotropic glutamate receptor 5 and glutamate involvement in major depressive disorder: a multimodal imaging study. Biol Psychiatry: Cogn Neurosci Neuroimaging. 2017;2:449-56

75. Njau S, Joshi SH, Espinoza R, Leaver AM, Vasavada M, Marquina A, et al. Neurochemical correlates of rapid treatment response to electroconvulsive therapy in patients with major depression. JPN. 2017;42:6-16.

76. Smith GS, Oeltzschner G, Gould NF, Leoutsakos J-MS, Nassery N, Joo JH, et al. Neurotransmitters and neurometabolites in late-life depression: a preliminary magnetic resonance spectroscopy study at 7T. J Affect Disord. 2021;279:417-25.

77. Williams JBW, Kobak KA, Bech P, Engelhardt N, Evans K, Lipsitz J, et al. The GRIDHAMD: standardization of the Hamilton Depression Rating Scale. Int Clin Psychopharmacol. 2008;23:120-9.

78. Kurtz M. The Penn Conditional Exclusion Test: a new measure of executivefunction with alternate forms for repeat administration. Arch Clin Neuropsychol. 2004;19:191-201.

79. Berg EA. A simple objective technique for measuring flexibility in thinking. J Gen Psychol. 1948;39:15-22.

80. Calhoun VD, Wager TD, Krishnan A, Rosch KS, Seymour KE, Nebel MB, et al. The impact of T1 versus EPI spatial normalization templates for fMRI data analyses: impact of T1 vs EPI spatial normalization templates. Hum Brain Mapp. 2017;38:5331-42.

81. Behzadi Y, Restom K, Liau J, Liu TT. A component based noise correction method (CompCor) for BOLD and perfusion based fMRI. Neurolmage. 2007;37:90-101.

82. Satterthwaite TD, Elliott MA, Gerraty RT, Ruparel K, Loughead J, Calkins ME, et al. An improved framework for confound regression and filtering for control of motion artifact in the preprocessing of resting-state functional connectivity data. Neurolmage. 2013;64:240-56.

83. Power JD, Barnes KA, Snyder AZ, Schlaggar BL, Petersen SE. Spurious but systematic correlations in functional connectivity MRI networks arise from subject motion. Neurolmage. 2012;59:2142-54.

84. Power JD, Cohen AL, Nelson SM, Wig GS, Barnes KA, Church JA, et al. Functional network organization of the human brain. Neuron. 2011;72:665-78.

85. Simpson R, Devenyi GA, Jezzard P, Hennessy TJ, Near J. Advanced processing and simulation of MRS data using the FID appliance (FID-A)-An open source, MATLAB -based toolkit. Magn Reson Med. 2017;77:23-33.

86. Provencher SW. Automatic quantitation of localizedin vivo1H spectra with LCModel. NMR Biomed. 2001;14:260-4.

87. Berrington A, Voets NL, Plaha P, Larkin SJ, Mccullagh J, Stacey R, et al. Improved localization for 2-hydroxyglutarate detection at $3 \mathrm{~T}$ using long-TE semi-LASER. Tomography. 2016;2:94-105.

88. Lindquist MA, Xu Y, Nebel MB, Caffo BS. Evaluating dynamic bivariate correlations in resting-state fMRI: a comparison study and a new approach. NeuroImage. 2014;101:531-46.

89. Choe AS, Nebel MB, Barber AD, Cohen JR, Xu Y, Pekar JJ, et al. Comparing testretest reliability of dynamic functional connectivity methods. Neurolmage. 2017;158:155-75

90. Fong AHC, Yoo K, Rosenberg MD, Zhang S, Li C-SR, Scheinost D, et al. Dynamic functional connectivity during task performance and rest predicts individual differences in attention across studies. Neurolmage. 2019;188:14-25.

91. Goldfarb EV, Rosenberg MD, Seo D, Constable RT, Sinha R, Hippocampal seed connectome-based modeling predicts the feeling of stress. Nat Commun. 2020;11: https://doi.org/10.1038/s41467-020-16492-2.

92. Shen X, Finn ES, Scheinost D, Rosenberg MD, Chun MM, Papademetris $X$, et al. Using connectome-based predictive modeling to predict individual behavior from brain connectivity. Nat Protoc. 2017;12:506-18.

93. Hengartner MP, Plöderl M. Statistically significant antidepressant-placebo differences on subjective symptom-rating scales do not prove that the drugs work: effect size and method bias matter! Front Psychiatry. 2018;9:517.

94. Stolk P, ten Berg MJ, Hemels ME, Einarson TR. Meta-analysis of placebo rates in major depressive disorder trials. Ann Pharmacother. 2003;37:1891-9.

95. Wijtenburg SA, Rowland LM, Oeltzschner G, Barker PB, Workman Cl, Smith GS. Reproducibility of brain MRS in older healthy adults at 7T. NMR Biomed. 2019;32:e4040.
96. Goldberg D. The heterogeneity of "major depression". World Psychiatry. 2011;10:226-8

97. Rosenberg MD, Zhang S, Hsu W-T, Scheinost D, Finn ES, Shen X, et al. Methylphenidate modulates functional network connectivity to enhance attention. J Neurosci. 2016;36:9547-57.

98. Rosenberg MD, Finn ES, Scheinost D, Papademetris $X$, Shen $X$, Constable RT, et al. A neuromarker of sustained attention from whole-brain functional connectivity. Nat Neurosci. 2016;19:165-71.

99. Rosenberg MD, Scheinost D, Greene AS, Avery EW, Kwon YH, Finn ES, et al. Functional connectivity predicts changes in attention observed across minutes, days, and months. Proc Natl Acad Sci USA. 2020;117:3797-807.

100. Cohen MR, Maunsell JHR. Attention improves performance primarily by reducing interneuronal correlations. Nat Neurosci. 2009;12:1594-1600.

\section{ACKNOWLEDGEMENTS}

This work was supported by the National Institute on Drug Abuse grants R01DA03889 (PI: RRG), T32DA007209 (PI: Bigelow), and a grant from the Heffter Research Institute. This work was also supported by the Steven and Alexandra Cohen Foundation, Tim Ferriss, Blake Mycoskie, Matt Mullenweg, Craig Nerenberg, the Riverstyx Foundation, and Dave Morin. The funders had no role in study design, data collection, and analysis, decision to publish, or preparation of the manuscript.

\section{AUTHOR CONTRIBUTIONS}

MKD conceptualized the analysis, analyzed the data, produced figures, and wrote the manuscript, MP analyzed the data, produced figures, and wrote the manuscript, MDR conceptualized the data analysis and wrote the manuscript, NDS collected the data, analyzed the data, and wrote the manuscript, AKD designed the study, collected the data, and wrote the manuscript, PHF designed the study and wrote the manuscript, GSS designed the study and wrote the manuscript, JJP designed the study, conceptualized the analysis, and wrote the manuscript, PBB designed the study and wrote the manuscript, RRG designed the study and wrote the manuscript, FSB designed the study, conceptualized the analysis, analyzed the data, produced figures, and wrote the manuscript.

\section{COMPETING INTERESTS}

MKD is on the scientific advisory board for Ocean Bio Limited. AKD is a board member of Source Research Foundation. PHF is on the scientific advisory board for and has an equity position in Ninnion Therapeutics. RRG is a board member of the Heffter Research Institute. FSB is an advisor to Wavepaths. All other authors have no competing interests to declare.

\section{ADDITIONAL INFORMATION}

Supplementary information The online version contains supplementary material available at https://doi.org/10.1038/s41398-021-01706-y.

Correspondence and requests for materials should be addressed to Manoj K. Doss.

Reprints and permission information is available at http://www.nature.com/ reprints

Publisher's note Springer Nature remains neutral with regard to jurisdictional claims in published maps and institutional affiliations.

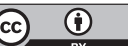

Open Access This article is licensed under a Creative Commons Attribution 4.0 International License, which permits use, sharing, adaptation, distribution and reproduction in any medium or format, as long as you give appropriate credit to the original author(s) and the source, provide a link to the Creative Commons license, and indicate if changes were made. The images or other third party material in this article are included in the article's Creative Commons license, unless indicated otherwise in a credit line to the material. If material is not included in the article's Creative Commons license and your intended use is not permitted by statutory regulation or exceeds the permitted use, you will need to obtain permission directly from the copyright holder. To view a copy of this license, visit http://creativecommons. org/licenses/by/4.0/.

(c) The Author(s) 2021 